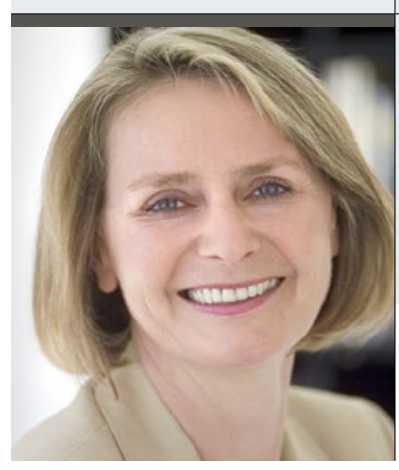

"Während in Diagnostik und Therapie bei Männern traditionell eher körperliche Ursachen im Fokus stehen, ist bei Frauen öfters eine Betonung der seelischen Komponente festzustellen."

Prof. Dr. med. Anette Kersting

Direktorin der Klinik und Poliklinik für Psychosomatische Medizin und

Psychotherapie, Universitätsklinikum Leipzig

\title{
Geschlechtsspezifische Aspekte mehr beachten!
}

E in Blick in repräsentative epidemiologische Erhebungen zeigt: Während Alkoholabhängigkeit und Suizid vermehrt Männer betreffen, werden bei Frauen häufiger Depressionen, Angstoder Essstörungen diagnostiziert. Unter den Betroffenen mit Depressionen oder Angststörungen ist der Anteil der Frauen im Vergleich zu den Männern doppelt so hoch, Magersucht betrifft sogar fast viermal so viele Frauen wie Männer. Ausnahmen sind die Zwangserkrankung und die bipolare Störung, bei denen Männer und Frauen gleich hohe Prävalenzraten zeigen [1].

Diese Statistiken spiegeln sich auch in den Arbeitsunfähigkeitstagen wider: Frauen fehlen am Arbeitsplatz deutlich häufiger aufgrund einer psychischen Erkrankung als ihre männlichen Kollegen. Nach aktuellen Daten der DAK-Gesundheit sind es im Geschlechtervergleich $67 \%$ mehr Fehltage. Hinzu kommt eine höhere Rate der Verschreibungen von Psychopharmaka: Jede elfte Frau bekam im vergangenen Jahr eine Verordnung für Antidepressiva, aber nur jeder zwanzigste Mann.

\section{Ursachen finden sich in der Kindheit ...}

Die Ursachen sind vielfältig und hängen mit Geschlechtsunterschieden in den Hirnstrukturen und -funktionen, Auswirkungen reproduktiver Hormone, aber auch mit der Responsivität auf Stress sowie mit unterschiedlichen sozialen Erwartungen und Erfahrungen zusammen. Geschlechtsunterschiede, die die Lebenserfahrungen und kulturellen Erwartungen betreffen, können die Genexpression beeinflussen und zur Entwicklung von Geschlechtsunterschieden im Gehirn und in der Physiologie während der Lebensspanne beitragen [2, 3]. Eine Herausforderung besteht darin $\mathrm{zu}$ verstehen, welche Geschlechtsunterschiede eine Relevanz für psychiatrische Erkrankungen haben [4]. Sexueller Missbrauch in der Kindheit, der bei Frauen häufiger auftritt als bei Männern, ist mit einem erhöhten Risiko für Depressionen und Angsterkrankungen während der Adoleszenz und im Erwachsenenalter verbunden [5]. Kindlicher Missbrauch geht darüber hinaus mit einer Dysregulation der biologischen Stressachse und Veränderungen der Gehirnentwicklung einher, die sich als Mediator für die affektiven Erkrankungen auswirken kann [6].

Ein anderer Geschlechtsunterschied, der sich auch in der Kindheit zeigt, sind Coping-Stile von Mädchen und Jungen. Geschlechtsbedingte Unterschiede der sozialen Unterstützung präsentieren sich bereits vor der Pubertät in der Sprache. Jungen neigen zu dominantem Verhalten, sie drohen und unterbrechen einander häufiger in gleichgeschlechtlichen Gruppeninteraktionen. Im Gegensatz dazu drücken Mädchen eher Übereinstimmung aus, sie akzeptieren die Argumentationsstrategien der anderen und pausieren in Gesprächen häufiger, um andere zu Wort kommen zu lassen. Weitere Studien zeigen, dass Jungen mehr vermeidende, Mädchen hingegen eher ängstliche Coping-Stile in persönlichen Beziehungen zeigen $[7,8]$.

\section{... und im Erwachsenenalter}

Diese Geschlechtsunterschiede setzen sich im Erwachsenenalter fort. In Situationen verstärkter Bedrohung zeigen Männer eher ein ausweichendes Verhalten oder versuchen, durch Aktivität die Situation zu bewältigen, während Frauen eher Unterstützung von anderen suchen [9]. Darüber hinaus sind Unterschiede in den Lebensbedingungen und der sozialen Lage von Bedeutung. Frauen sind häufiger alleinerziehend und gehören, auch deshalb, häufiger niedrigen Einkommensschichten an. Die Vereinbarkeit von Beruf und Kindererziehung sowie die Betreuung pflegebedürftiger Angehöriger, die ebenfalls häufiger durch Frauen erfolgt, sorgen für zusätzliche Belastungen. Chronischer Stress 


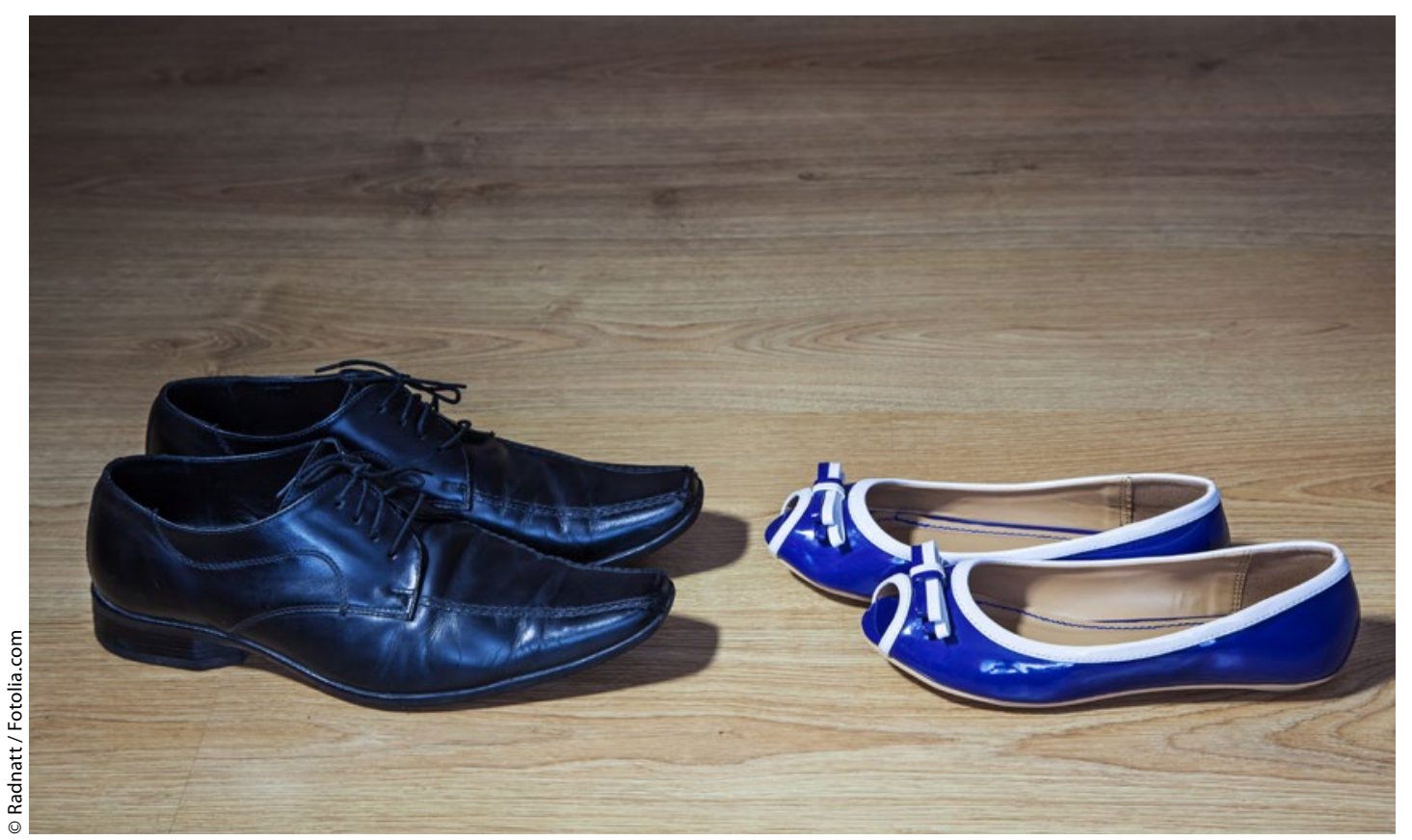

Es besteht noch Forschungsbedarf. Aber auch das bereits vorhandene Wissen muss verstärkt in die Versorgung einbezogen und die spezifischen weiblichen und männlichen Aspekte psychischer Erkrankungen müssen berücksichtigt werden.

und psychische Erkrankungen können die Folge sein. Auch frauenspezifische Lebensphasen wie die Zeit vor und nach der Geburt eines Kindes oder auch die Menopause erhöhen die Wahrscheinlichkeit für ein psychisches Ungleichgewicht.

Während bei Männern in der Diagnostik und Therapie traditionell eher körperliche Ursachen im Fokus stehen, ist bei Frauen eine Tendenz zur Betonung der seelischen Komponente festzustellen. Auf die geschlechtsspezifischen Aspekte bei der Therapie unipolarer Depressionen gehen Juliane Gruber und Michael Grube in ihrem CME-Beitrag in dieser Ausgabe (Seite 48) näher ein.

Grundsätzlich besteht noch großer Forschungsbedarf. Aber auch mit dem bereits vorhandenen Wissen besteht eine große Notwendigkeit, diese auch in die Versorgung einzubeziehen und die spezifischen weiblichen und männlichen Aspekte psychischer Erkrankungen zu berücksichtigen. Das betrifft die unterschiedlichen Zugangsregelungen zu den Hilfsangeboten ebenso wie die Gesundheitsfürsorge und das betriebliche Gesundheitsmanagement. Unser Wissen sollte dafür genutzt werden, die Maßnahmen noch gezielter an den individuellen Bedürfnissen der Betroffenen auszurichten.

Herzliche Grüße

\section{Literatur}

1. Altemus M, Sarvaiya N, Epperson N. Frontiers in Neuroendocrinology 2014; 35: 320-30

2. Curley J, Jensen C, Mashoodh R et al. Psychoneuroendocrinology 2011; 36: 352-71

3. Springer K, Stellman J, Jordan-Young R. Soc Sci Med 2012; 74: $1817-24$

4. DeVries G, Sodersten P. Horm Behav 2009; 55: 589-96

5. Felitti V, Anda R, Nordenberg D et al. J Am J Prev Med 1998; $14: 245-58$

6. DeBellis M, Spratt E, Hooper S. J Child Sex Abus 2011; 20 (5): $548-87$

7. Carter R J. Clin Child Adolesc Psychol 2011; 40: 730-41

8. Gluck R, Lynn D, Dritschel et al. Br J Dev Psychol 2014; 13 Epub

9. Taylor S, Klein L, Lewis B et al. Psychol Rev 2000; 107: 411-29
Professor Dr. med. Anette Kersting

Leiterin des DGPPN-Referats Frauen- und geschlechtsspezifische Fragen in der Psychiatrie

Klinik und Poliklinik für Psychosomatische Medizin und Psychotherapie,

Universitätsklinikum Leipzig AöR

Semmelweisstr. 10, 04103 Leipzig

E-Mail: MB-PSOM-Sekretariat@medizin.uni-leipzig.de 